\title{
Optimal Energy-Efficient Routing for Wireless Sensor Networks
}

\author{
Chih-Wei Shiou, Frank Yeong-Sung Lin, Hsu-Chen Cheng, and Yean-Fu Wen \\ Department of Information Management, National Taiwan University (NTU) \\ \{r91044;yslin;d7725002; d89002\}@im.ntu.edu.tw
}

\begin{abstract}
The network lifetimefor wireless sensor network plays an important role to survivability, Thus, we indicate the importance of routing protocol to network lifetime, and model the expected retransmission time as a convex function with respect to aggregate jlow on each sensor node. Thus we formulate the optimal energy-eficient routing as a non-linear min-max programming problem with convex product form, which can be optimally solved by optimal routing framework. Based on the optimal routing framework, we propose Lagrangean-based algorithm and primal optimal algorithm. By the combination of these two algorithms, we can optimally and efficiently get the routing assignment to maximize the network life in the sensor network. From experiments, we observe that when the optimal network lifetime increases as the number of sensor nodes increase. While the shortest path-based heuristic algorithm can only achieve about $48 \%$ network lifetime compared to our solution approach.
\end{abstract}

\section{Introduction}

In the wireless sensor network, the energy aware routing (EAR) protocol was presented to extend the network life time [4] [18]. The EAR requires hardware support which is the capability of knowing the battery status, e.g., how many watts the node still remains. In [3], this concept was enhanced by introducing "altruist", the node having surplus energy to forward traffic, into wireless sensor networks. Through properly exchanging battery status between neighbors, the routing policy is energy efficient and the network life time extends significantly.

Since there are only a few OD (Original Destination) pairs needed to be recorded in each node's routing table, we can compute several candidate routing paths for each OD pair. The benefit of multiple candidate routing paths was argued in [11]. We apply the features of optimal routing [14] when selecting the candidate paths and scaling traffic flow between paths until the optimality conditions are satisfied.

In this paper, we choose table-driven routing policy and apply distance vector based algorithm, e.g. distributed Bellman-Ford (DBF) algorithm. To take the advantage of the asynchronous convergence property of DBF [7], we build a routing protocol implemented with distributed fashion which is indispensable in practice for sensor networks. To apply optimal routing features on DBF, we have to define the link length as sophisticated parameters which are capable of affecting network life time as in [1] and [16].

Based the analysis of the expected retransmission time and the collision probability in [9], we model the expected retransmission time as a convex function of aggregate traffic load on the node then take it into the proposed routing algorithm. In [5], the author proposed that min-max node lifetime objective function tends to find longer path resulting to decrease average node lifetime. arr algorithm contributes to keep balance between minimum node lifetime and average node lifetime in this stage.

The sensor deployment component is for topology determination [6]. Note that the sensor network topology is non-regular and usually randomly spread as [8]. We formulate the energy efficient routing problem as a nonlinear optimization problem. To fulfill the timing and the quality of the optimal decisions, the solution approach to the mathematical problem is Lagrangean relaxation method. In the further computational experiments, our proposed routing algorithm is expected to be efficient and effective to deal with each complexity problems.

The remainder of this paper is organized as follows. In Section 2, we briefly describe the optimal energy routing problem and present the problem formulation. In Section 3 , the solution approach is presented. In Section 4, illustrate algorithms to solve the optimal mathematical problem and compare with other methods with experimental results in section 5. Finally, we present our conclusions in Section 6.

\section{Problem description}

In sensor networks, the interference is a significant effect on communication, which will affect bit-error rate and retransmission. To get an over-estimated retransmission model, consider the pure-aloha MAC formulation which can be taken as a performance lower-bound of those MAC layers in practice:

$$
h=G e^{-2 G}
$$

here $\bar{h}$ is the throughput of the transmitter node, defin (1) aggregate flow divided by wireless channel capacity. 
Notation $\mathrm{G}$ is the traffic load including retransmission for the transmitter node. Then the expected retransmission time $\mathbf{R}$ is:

$$
\mathrm{R}=E[\text { retran_time }]=\sum_{\mathrm{k}=\mathrm{l}}^{\infty} k P_{s}\left(1-P_{s}\right)^{k-1}=e^{2 \mathrm{G}}
$$

where $P_{s}$ is the successful transmission probability. From the deduction in [2], the expected retransmission time in pure-Aloha system is $e^{2 G}$. Give the deduction as following, first we apply Tyler expansion at $\mathrm{G}=\mathrm{O}($ then $R=I$ ) on $f(R)$ $=\ln (R)$ :

$$
h=\frac{\ln R}{2 R}=-\frac{R}{4}+1-\frac{3}{4 R}
$$

After applying the quadratic equation formula, the expected retransmission time $\mathbf{R}$ is a function of throughput h:

$$
\left\{\begin{array}{cc}
R(h)=2-2 h-\sqrt{4 h^{2}-8 h^{+}} & \text {if } h \leq 0.133 \\
R(h)=R(0.133)+R^{\prime}(0.133)(h-0.133) & \text { if } h>0.133
\end{array}\right.
$$

Then, we develop a mathematical model to deal with the energy efficient routing problem in order to maximize the network life time in the system. Table 1 lists the given parameters and Table $\mathbf{2}$ lists the decision variables. According the above discussions, we formulate the energy efficient routing problem as a complex nonlinear programming.

Objective function:

$$
\mathrm{Z}=\max \min _{n} t_{n}
$$

Subject to:

$$
\begin{aligned}
& \sum_{w e w} r_{t w}=R_{i} \\
& \sum_{p \in P_{w}} f w p=\sum_{i \in V} r_{i w} \\
& \forall w \in W \\
& \sum \sum f_{w p} \delta_{n p}=g_{n} \\
& \forall n \mathbf{E} \mathbf{N} \\
& \text { wew } p \in P_{w} \\
& h_{n}=g_{n} / c_{n} \\
& \forall n \in \mathbf{N} \\
& 2 g_{n} \leq\left(1-q_{n}\right) c_{n} \\
& \forall n \mathbf{E} \mathbf{N} \\
& R\left(h_{n}\right)=2-2 h_{n}-\sqrt{4 h_{n}^{2}-8 h_{n}+1} \quad \forall n \in N, h_{n} \leq 0.133 \\
& R\left(h_{n}\right)=1.6518+40.1924\left(h_{n}-0.133\right) \forall n \in N, h_{n}>0.133 \\
& \frac{E_{n}}{g_{n} R\left(h_{n}\right) e_{n}+a_{n}\left(1-q_{n}\right)+d_{n} q_{n}}=t_{n} \quad \forall n \in \mathbf{N} \\
& 0 \leq q_{n} \leq 1 \\
& \forall n \in \mathbf{N} \\
& f_{w p} \geq 0 \\
& \forall w \in W, p \in P_{w} \\
& f_{w p}=0 \\
& r_{i w} \geq 0 \\
& r_{i \bar{w}}=0 \\
& \begin{array}{r}
\forall \bar{w} \in\{N-W\}, p \in P_{w} \\
\forall w \in W, i \in V \\
\forall \bar{w} \in\{\mathrm{N}-W\}, i \in V
\end{array}
\end{aligned}
$$

The objective function is to maximize the network lifetime of the given wireless sensor network configuration. The network lifetime is related to the routing policy and passive mode management, which are
Table 1. Notation descriptions for given parameters

\begin{tabular}{|c|l|}
\hline Notation & \multicolumn{1}{|c|}{ Descriptions } \\
\hline$N$ & The set of wireless sensor nodes; \\
\hline$V$ & $\begin{array}{l}\text { The set of events subscribed by the application layer } \\
\text { services in the sensor network; }\end{array}$ \\
\hline$W$ & $\begin{array}{l}\text { The node set being capable of sensing subscribed } \\
\text { events; }\end{array}$ \\
\hline$D$ & The set of specific destination nodes; \\
\hline$R_{i}$ & $\begin{array}{l}\text { The traffic flow source from subscribed event } i \\
\text { measured by Kbps (kilobytes per second), assuming } \\
\text { it is constant bit rate; }\end{array}$ \\
\hline$P_{w}$ & $\begin{array}{l}\text { The candidate paths to the destination and origin } \\
\text { from node } w, w \in W ;\end{array}$ \\
\hline$E_{n}$ & The initial energy level of node $n$, measured by Watt; \\
\hline$e_{n}$ & $\begin{array}{l}\text { The transmission energy required by node } n \text { to } \\
\text { transmit an information unit, measured by Watt per } \\
\text { kilobyte; }\end{array}$ \\
\hline$c_{n}$ & The capacity on node $n$, measured by Kbps; \\
\hline$a_{n}$ & $\begin{array}{l}\text { The energy required by node } n \text { to retain active mode, } \\
\text { measured by Watt per second; }\end{array}$ \\
\hline$d_{n}$ & $\begin{array}{l}\text { The energy required by node } n \text { to retain passive } \\
\text { mode, measured by Watt per second; }\end{array}$ \\
\hline$R\left(h_{n}\right)$ & $\begin{array}{l}\text { The expected retransmit time until success. It is a } \\
\text { convex function related to the channel throughput of } \\
\text { node } n \text {, measured by kilobytes per second. }\end{array}$ \\
\hline$\delta_{\mathrm{np}}$ & $\begin{array}{l}\text { Indicator function which is a } 0-1 \text { variable. If node } n \text { is } \\
\text { in path } p \text { then set to 1, otherwise 0; }\end{array}$ \\
\hline
\end{tabular}

Table 2. Notation descriptions for decision variables

\begin{tabular}{|c|l|}
\hline Notation & \multicolumn{1}{|c|}{ Descriptions } \\
\hline$r_{i w}$ & $\begin{array}{l}\text { The traffic flow source from subscribed event } i \text { and } \\
\text { sensed by node } w, \text { measured by measured by Kbps. }\end{array}$ \\
\hline$f_{w p}$ & $\begin{array}{l}\text { The traffic flow source from node } w \text { and route through } \\
\text { path } p, \text { measured by measured by Kbps; }\end{array}$ \\
\hline$g_{n}$ & Aggregate flow on node $n ;$ \\
\hline$h_{n}$ & Channel throughput of node $n ; h_{n}=g_{n} / c_{n}$ \\
\hline$q_{n}$ & $\begin{array}{l}\text { The portion that node } n \text { is in passive mode of it self's } \\
\text { node life time. It is in [0, 1]. }\end{array}$ \\
\hline$t_{n}$ & The time duration of node $n$ to exhaust its energy; \\
\hline
\end{tabular}

the decision variables in our formulation. Constraint (5) ensures that the event-driven traffic can be fully dispatched to the corresponding sensors. Constraint (6) is the path-oriented routing requirement constraint. Constraint (7) calculates the aggregate flow on node $n$. Constraint (8) calculates the channel throughput according to the aggregate flow on node $n$. Constraint (9) is bandwidth constraint on wireless sensor nodes. Constraints (10) and (11) are both convex functions modeling the expected number of retransmission time related to the channel throughput of node n. Constraint (12) calculates the node lifetime concerning the aggregate traffic flow on node $n$, the energy consumption rate and the frequency that node $\mathrm{n}$ is in passive mode. Constraint (13) enforces the portion that node $n$ is in passive mode of its node lifetime is between 0 and 1. Constraints (14)-(17) ensure the traffic flow are positive or zero.

Because the node lifetime $t_{n}$ must be positive, the original objective function can be rewritten as following:

$$
Z=\max \min _{n} t_{n}=\min \max _{n}\left(1 / t_{n}\right)
$$




$$
=\min \max _{n}\left[g_{n} R\left(h_{n}\right) e_{n}+a_{n}\left(1-q_{n}\right)+d_{n} q_{n}\right] / E_{n}
$$

Also, at the optimum, the passive mode must be fully utilize to achieve the best energy-efficient. Constraint (9) is active andq, $=1-2 g_{n} / c_{n}$. Thus an equivalent formulation of Problem (PB1) is:

Objective function:

$$
Z=\min \max _{n} \frac{g_{n} R\left(h_{n}\right) e_{n}+\left(a_{n}-d_{n}\right) \frac{2 g_{n}}{c_{n}}+d_{n}}{E_{n}}
$$

Subject to: (5) - (17) except (9), (12), and (13).

This re-formulation eliminates Constraints (9), (12), and (13) as well as decision variables $q_{n}$ by merging them into the objective function and the problem becomes a single decision variable programming problem.

\section{Solution approach}

The optimal energy-efficient routing problem (OEERP) in wireless sensor networks is a nonlinear programming problem with convex product form. We apply Lagrangean Relaxation (LR), which has been successfully adopted to solve many famous NP-complete problems [13], to solve the optimal energy-efficient routing problem. By Lagrangean strong duality theorem, the tightest lower bound attained by Lagrangean dual problem is exactly the primal feasible objective function [15]. We also conduct a primal algorithm to get the optimal routing assignment resulting to maximize network lifetime.

We transform the maximization problem to minimization without loss of correctness.

Let $s=\max _{n \in N}\left[g_{n} R\left(h_{n}\right) e_{n}+2 g_{H}\left(a_{n}-d_{n}\right) / c_{n}+d_{n}\right] / E_{n}$, then an equivalent formulation of Problem (PB2) is:

Objective function:

$$
Z=\operatorname{mins}
$$

Subject to:

$$
\begin{aligned}
& \text { (5) }-(17) \text { except (9), (12), and (13). } \\
& \mathrm{s}>\mathrm{O} \\
& g_{n} R\left(h_{n}\right) e_{n}+2 g_{n}\left(a_{n}-d_{n}\right) / c_{n}+d_{n} \leq s E_{n} \quad \mathrm{Vn} \in \mathrm{N}
\end{aligned}
$$

Constraint (19) ensures the equality with original problem (PB1), Constraint (20) defines the minimum node lifetime in the network. By using the LR method, the primal problem can be transformed into the following LR problem where Constraint (20) is relaxed. For a vector of non-negative Lagrangean multipliers, the LR problem is given by optimization problem as:

Objective function:

$$
\begin{aligned}
& Z_{L R}(\alpha)=\min s+ \\
& \sum_{n \in N} \alpha_{n}\left[g_{n} R\left(h_{n}\right) e_{n}+2 g_{n}\left(a_{n}-d_{n}\right) / c_{n}+d_{n}-s E_{n}\right]
\end{aligned}
$$

Subject to: (5) (19) except (9), (12), (13) and (18).

In this formulation, $a$ is the vector of $\left\{\alpha_{n}\right\}$, which are Lagrangean multipliers and $a, \geq 0$. To solve this problem,
Subproblem (SUB2): related with decision variables $r_{i w}$ and $f_{w p}$.

Subiect to: (5) (7), (10) (11), and (14) (17).

Subproblem(SUB2-2): related with decision variables $x_{i p}$. Objective function:

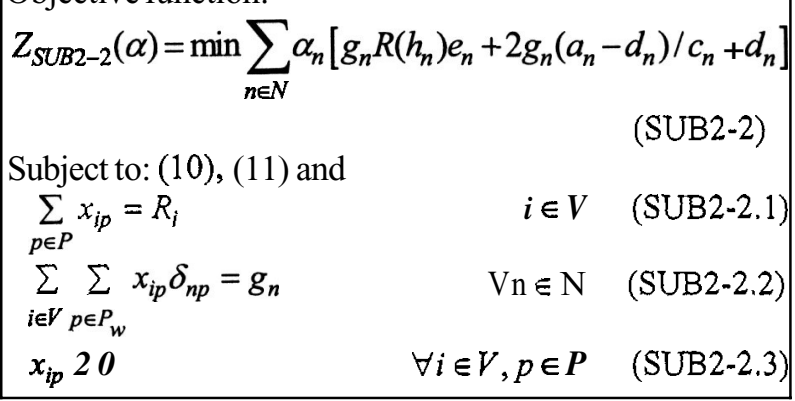


vector $\pi$ is updated by $\pi^{k+1}=\pi^{k}+\xi^{k} \chi^{k}$. The step size $\xi^{k}$ is determined by $\zeta^{k}=\zeta \cdot Z^{h}-Z_{D}\left(\pi_{k}\right)\left\|\chi^{k}\right\|^{2}$, where $Z^{h}$ is the primal objective function value for a heuristic. It is an upper bound on $Z_{D}$.

We describe the solution procedure of optimal energy-efficient routing as Fig. 1 and the detailed description of each procedure is:

a. Computing First Derivative Length

By definition, the cost function of Subproblem (SUB2-2) that the object is to minimize the sum of reciprocal of node lifetime weighted by Lagrangean multipliers, and the FDL is the first derivation with path flow. We get the first derivative length as following:

$$
\sum_{n \in N} \alpha_{n}\left[R\left(h_{n}\right) e_{n}+g_{n} R^{\prime}\left(h_{n}\right) \frac{d h_{n}}{d g_{n}} e_{n}+\frac{2\left(a_{n}-d_{n}\right)}{c_{n}}\right]
$$

Given the valuation of first derivative length as mentioned above, at every iteration we compute each node's lifetime according the current routing assignment, and recognize the bottleneck node in the network. Then update the first derivative length of all the paths. Finally we shift flow between paths based on the first derivative length computed earlier. Repeat these steps until algorithm converge.

b. Finding MFDL Path

Our goal is to find the most economic path to shift positive flow iteration by iteration. By the lemmas of optimal routing framework, the most economic and energy-efficient path must with the minimum sum of Node-FDL. Thus we directly apply Dijkstra shortest path algorithm to find the MFDL path for each OD-pair iteration by iteration, where the length computed for shortest path is the node-FDL.

\section{c. Path Flow Adjustment}

We shift positive amount of flow form the maximum FDL path to the minimum one by applying Newton method on line search. Let $\beta^{*}$ be the step size that minimizes $D\left[x+\beta\left(x^{\prime}-x\right)\right]$ over all $\beta$ is between 0 and 1 , that is,

$$
D\left[x+\beta^{*}\left(x^{\prime}-x\right)\right]=\min _{\beta \in[0,1]} D\left[x+\beta^{*}\left(x^{\prime}-\mathbf{x}\right)\right]
$$

The procedure above is a special case of the so-called Frank-Wolfe method for solving nonlinear programming

\begin{tabular}{|c|c|}
\hline ep 1: & $\begin{array}{l}\text { hs of each OD-pairs and init any arbitrary } \\
\text { ng assignment set. }\end{array}$ \\
\hline ep 2: & $\begin{array}{l}\text { Stopping. If } k \text { is greater than a pre-specified counter limit then } \\
\text { stop. }\end{array}$ \\
\hline tep 3: & $\begin{array}{l}\text { Computing. Update the aggregate flow on each node in the } \\
\text { network according to the currentrouting assignment. }\end{array}$ \\
\hline Step 4 & $\begin{array}{l}\text { Updating. Compute node-FDL and path-FDL according to the } \\
\text { up-to-date aggregate flow. }\end{array}$ \\
\hline ep 5: & $\begin{array}{l}\text { Shiftiig Flow. Shift flow between paths according to } \\
\text { path-FDL. Increase } k \text { by } 1 \text { and go to Step } 2 \text {. }\end{array}$ \\
\hline
\end{tabular}
problems with convex constraint sets.

Figure 1. An optimal energy-efficient routing algorithm

\section{Energy-efficient routing algorithm}

The primal optimal algorithm is similar with that we use to solve Lagrangean Subproblem (SUB2). However, the procedures including computing path FDL phase, finding path phase, and flow adjustment phase are different. This difference is resulted from the min-max behavior and the differential property. We will give a detailed description of min-max Dijkstra algorithm as followings:

\section{a. Finding Minimum Capacity Cut Path}

Because the objective function in our primal problem is a min-max function, but the general Dijkstra shortest path algorithm is not suit for finding MFDL path. Thus, we modify the Dijkstra algorithm to find such a min-max shortest path and the pseudo code is showed as Figure 2.

The Single-Source-Minimum-Cut-Paths (SSMCP) algorithm finds the path with minimum capacity cut form the given OD-pair. However, this algorithm only returns the minimum cut capacity value but does not record the path. Figure 3 shows an example of two paths with the same capacity cut.

The two paths are with the same minimum capacity cut which value is c. In general, we choose the right path with fewer hops as a better energy-efficient path than

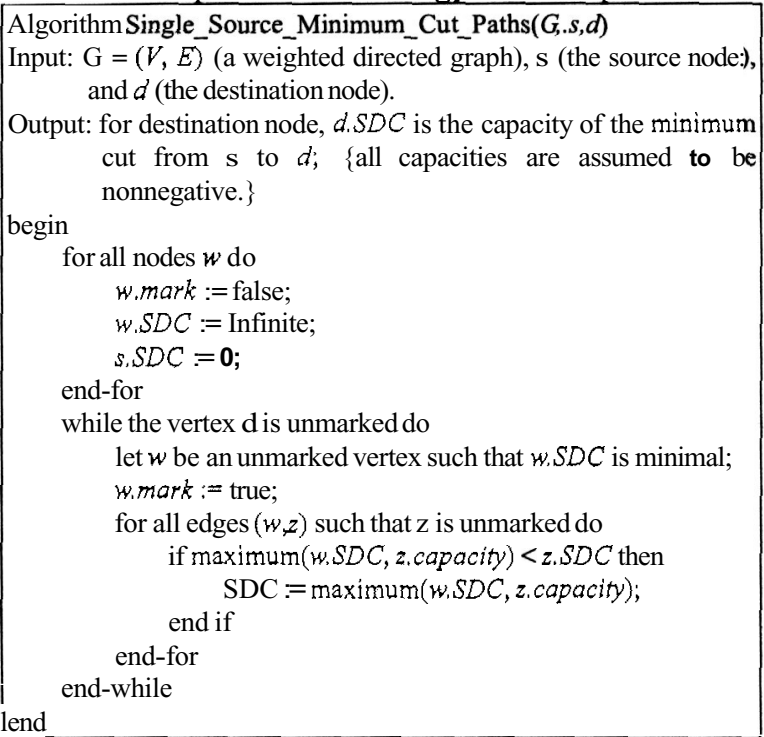

Figure 2. Single-Source-Minimum-Cut-Path algorithm
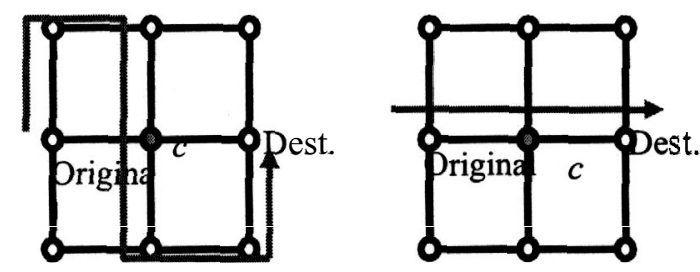

Figure 3. Example for two paths with the same capacity cut 
the left. But the SSMCP algorithm may still possible return the left path. So based on the output of SSMCP algorithm, we apply modified Dijkstra algorithm to find the shortest and minimum cut path. Figure 4 shows the pseudo-code of Capacity- Constrained-Shortest-Path (CCSP) is for this purpose.

\section{b. Polya's Method on Path Flow Adjustment}

The basic idea to adjust path flow as the bottleneck node lifetime of path $p$ is the minimum in the sensor network, and then we decrease the fraction of path flow o n p according to specific step sizes and add back the same amount of flow to another path with larger bottleneck node lifetime. The step of flow adjustment procedure is given as Figure 5.

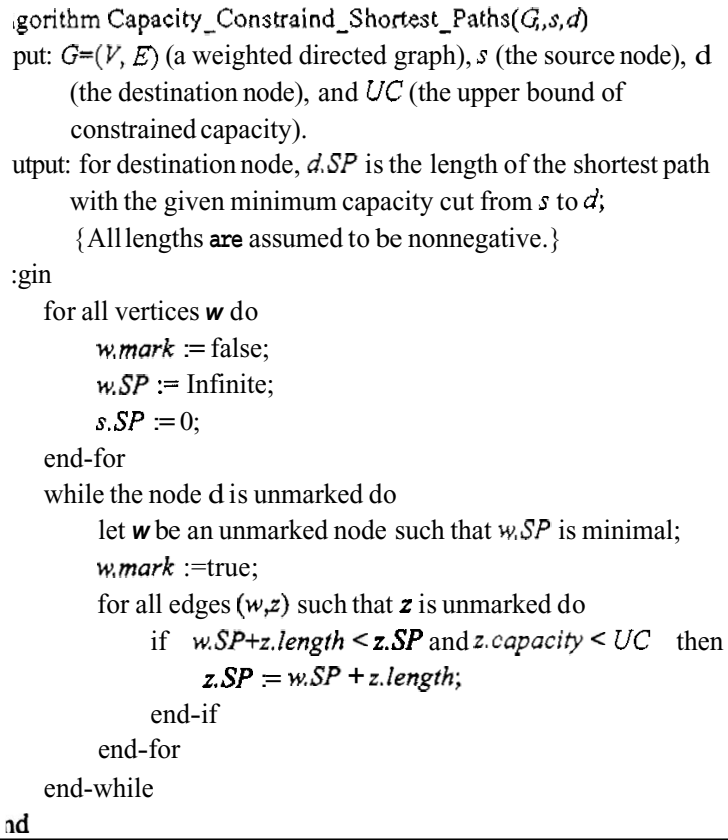

Step 1: Initialization. Set the iteration counter $\boldsymbol{k}$ to be 1. Compute the path bottleneck lifetime according to any given feasible routing assignment.

Step 2: Stopping. If $\boldsymbol{k}$ is greater than a pre-specified counter limit then stop.

Step 3: Finding. Find the paths with minimum and maximum bottleneck lifetime.

Step 4: Selection. Select path with minimum bottleneck lifetime and denote its flow $f$. Shift the fraction of $f$ by a positive stepsize $t_{f}^{k}$. More precisely, we shift flow with amount of $f^{*} s_{f}^{k}$ form the path with minimum bottleneck lifetime

\section{Experimental results}

The experimentation variable $\delta$ is defined as the ratio between the edge lengths of grid area and the sensor's communication radius. The experimental result is given as Table 3. In wireless sensor networks, the communication radius is about 12.5 meters and when $=8$ the area size is $100 \cdot 100$ meters. We adopt the energy consumption parameter of EYES-nodes [19] in our study. For each sensor node, the parameters are as follows:

- Wireless channel capacity is $10 \mathrm{kbps}$.

- Initial battery capacity of each sensor node is between 1300 and 1600 Watts.

- Energy consumption rate on receiving (transmitting) is 0.2 Watts per byte.

- Energy consumption rate to retain in active mode (passive mode) is 50 (10) Watts per second respectively.

In our computational experiments, we generate several system scenarios with different (1) average package length and (2) sensor node density. Then we apply the primal optimal algorithm introduced in Section 3 and Section 4 to compute the maximum network lifetime.

To experiment (1) average package length, we set up two cases with different parameters. In Case 1, the area size ratio $-\bullet$ is 4 . Here we set the number of nodes in Case 1 is 27 , which is 1.5 times the minimum number of sensor nodes from Table 3 . And traffic demand is set as 5 which is 0.2 times the number of sensor nodes. In Case 2, the area size ratio. - is 8 , and we set up parameters according to the 'same logic as in Case 1. The experimental results are given in Table 4.

To experiment (2) sensor node density, we set up two cases with different parameters. In Case 3 , the area size ratio - • is 4. Here traffic demand is fix at 6 and average packet length is 200 bytes. The numbers of nodes are 1.5, 2.5, 3.5, and 4.5 times the minimum number of sensor nodes satisfying 1-connectivity. So they are $27,45,63$, and 81 respectively. In Case 4, the area size ratio- - is 8 . Those parameters in both Cases are list below, and the experimental results are given in Table 5 .

In Table 4, we evaluate the effect of the expected retransmission time function which is consistent with our convex function to the aggregate flow on each sensor node Table 5 shows how the connectivity and the number of sensor node affect the network lifetime. It is clear that if the number of sensor nodes increases, it is with higher probability the network topology has higher connectivity. Thus the average network lifetime increase.

Figure 6 shows the network lifetime comparison between our primal optimal algorithm (PO) and Short-based algorithm (SP), which adopt the Dijkstra algorithm. Even though the path is the shortest path from the point of view with node lifetime and energy-efficiency, it is still fragile if any one bottleneck node exhaust it battery life. While our PO algorithm use multiple paths to route traffic between every OD-pairs, and the network 
lifetime increase as the number of sensor nodes increases.

We measure the primal optimal algorithm with time-complexity. The $|N|,|W|$, and $|P|$ denote the number of sensor nodes, the number of OD-pairs, the number of candidate paths of each OD-pair, respectively. Table 7 shows the number of operations required and time complexity of optimal routing framework for one iteration.

\section{Conclusions}

In this paper, we address the importance of routing protocol on energy efficiency, arr proposed LR-based algorithm can efficiently get the near-optimal solution and the primal algorithm can optimally solve the problem but spending much more time. Both of them are variations of optimal routing framework, which is to optimally solve the multi-commodities routing problem. We appraise the marginal cost of candidate paths by MFDL in LR-based algorithm and by capacity cut in primal optimal algorithm respectively. Using the quantity we adjust flow between paths for each OD-pair until the two optimal conditions are satisfied. Then we eventually achieve the optimal routing which is energy-efficientweighted load-balancing.

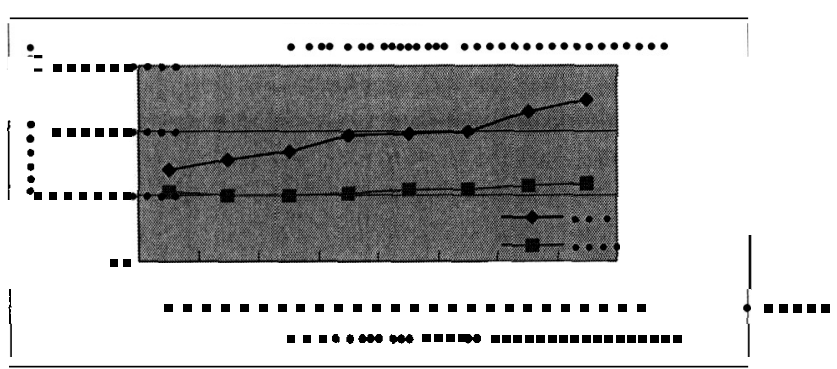

Figure 6. Comparing network lifetime between PO and SP

Table 3. Minimum number of sensor nodes to achieve 1-connectivity by different ratio $\delta$

\begin{tabular}{|c|c|c|c|c|}
\hline Ratio - . & 4 & 8 & 12 & 16 \\
\hline Number of sensors & 18 & 131 & 477 & 881 \\
\hline
\end{tabular}

Table 4. Experimental results for average packet lennth

\begin{tabular}{|c|c|c|}
\hline \multicolumn{2}{c}{} & \\
\hline 100 & 14925 & 665.9 \\
\hline 300 & 4075 & 50.4 \\
\hline 500 & 844 & 17.0 \\
\hline
\end{tabular}

Table 5. Experimental results for sensor node number

\begin{tabular}{|c|c|c|}
\hline \multirow{2}{*}{ Sensornodes } & \multicolumn{2}{c|}{ Network lifetime } \\
\cline { 2 - 3 } & Case 3 & Case 4 \\
\hline 27 & 23194 & 123 \\
\hline 45 & 23901 & 418 \\
\hline 63 & 28544 & 1035 \\
\hline \multicolumn{2}{|c}{} \\
\hline
\end{tabular}

Table 6. The time complexity of optimal routing framework

\begin{tabular}{|c|c|c|}
\hline \multicolumn{3}{|c|}{ Optimal routing framework } \\
\hline Procedure & $\begin{array}{c}\text { Number of operations } \\
\text { required }\end{array}$ & Time complexity \\
\hline Updating node flow & $|N|+|W||P||N|$ & $O(W|| P \| N \mid$ \\
\hline Finding MFDL path & $|W||N|^{2}$ & $O\left(\left.|W| N\right|^{2}\right)$ \\
\hline Computing path FDL & $|W||P||N|$ & $O(|W| P|| N \mid)$ \\
\hline Flow adjustment & $|W|^{*}(|P|+1)$ & $O(|W||P|)$ \\
\hline
\end{tabular}

\section{References}

[1] Hac A., Wireless Sensor Network Designs, John Wiley \& Sons, Ltd. 2003.

[2] Tanenbaum A. S., Computer Networks, Fourth Edition, Prentice-Hall, 2002.

[3] A. Willig, R. Shah, J. Rabaey and A. Wolisz, "Altruists in the PicoRadio Sensor Network", International Workshop on Factory Comm. Systems (WFCS), 2002.

[4] Perkins C. E., Ad Hoc Networking, Addison-Wesley, 2000.

[5] C. K. To, "Maximum Battery Life Routing to Support Ubiquitous Mobile Computing in Wireless Ad hoc Networks", IEEE CommunicationsMagazine, 2001.

[6] C. Schurgers, V. Tsiatsis, S. Ganeriwal and M. Srivastava, "Optimizing Sensor Networks in the Energy-Latency-Density Design Space”, IEEE Tran. on Mobile Computing, 1(1), 2002.

[7] Bersekas D. and R. Gallager, Data Networks, Prentice-Hall, 2nd edition, 1992,pp. 404

[8] E. Biagioni and K. Bridges, "The Application of Remote Sensor Technology to Assist the Recovery of Rare and Endangered Species", Special issue on Distributed Sensor Networks for the International Journal of High Performance Computing Applications, $16(3), 2002$.

[9] G Bianchi, "Performance Analysis of the IEEE 802.11 Distributed Coordination Function", IEEE J. Select. Areas Comm. ,vol.18, 2000, pp.318-320.

[10] G Zussman and A. Segall, "Energy Efficient Routing in Ad Hoc Disaster Recovery Networks", Proc. of IEEE INFOCOM 2003.

[11] J. J. Garcia-Luna-Aceves, S. Vutukury and W. T. Zaumen, "A Practical Approach to Minimizing Delays in Intemet Routing," IEEE ICC'99, 1999.

[12]M. Held, P. Wolfe and H. P. Crowder, "Validation of Subgradient Optimization," Math. Programming, vol.6, 1974,pp.62-88.

[13]M. L. Fisher, "The Lagrangian Relaxation Method for Solving Integer Programming Problems", Management Science, 27(1), 1981, pp.1-18.

[14]R. Gallager, "A Minimum Delay Routing Algorithm Using Distributed Computation", IEEE Trans. Comm., vol,25, 1977, pp.73-84.

[15]R. L. Cruz, and A. V. Santhanam, "Optimal Routing, Link Scheduling and Power Control in Multi-hop Wireless Networks", Proc. of IEEE INFOCOM 2003.

[16] R. Kannan, S. Sarangi, S. S. Iyengar and L. Ray, "Sensor-Centric Quality of Routing in Sensor Networks", Proc. of IEEE INFOCOM 2003.

[17]Ahuja R. K., T. L. Magnanti and J. B. Orlin, Network Flows: Theory, Algorithms, and Applications, Prentice Hall, 1993.

[18]R. C. Shah and J. M. Rabaey, "Energy Aware Routing for Low Energy Ad Hoc Sensor Networks", Proc. of IEEE Wireless Comm. and networking Conference (WCNC), 2002.

[19] T. V. Dam, and K. Langendoen, "An Adaptive Energy-Efficient MAC Protocol for Wireless Sensor Networks", Proc. of The First ACM Conference on Embedded Networked Sensor Systems, 2003. 